\title{
PELVIC LIPOMATOSIS IN AN ASYMPTOMATIC ASIAN MAN: A CASE REPORT
}

\section{Ng KL, Lee G}

Division of Urology, Department of Surgery, Faculty of Medicine, University of Malaya, Kuala Lumpur, Malaysia.

\begin{abstract}
:
Pelvic lipomatosis is a rare disease of unknown cause, characterised by overgrowth of benign mature fat cells commonly located in the perivesical and perirectal spaces. The disease is rare amongst Asian population and has a wide range of presenting symptoms varying from lower urinary tract symptoms to bowel symptoms. However, this disease can be easily diagnosed based on certain characteristic radiological imaging techniques. We report a case of pelvic lipomatosis in an asymptomatic Chinese gentleman, who presented with classical radiological features of pelvic lipomatosis but provided us with challenging therapeutic scenario. (JUMMEC 2010; 13(2): 111-114)
\end{abstract}

KEYWORDS: pelvic lipomatosis, perivesical, perirectal, Asian

\section{Case report}

A 46-year old overweight Chinese man presented to the University of Malaya Medical Centre (UMMC) in the middle of 2008 with microscopic haematuria following routine health screening at another centre. There were no significant urinary symptoms and risk factors for haematuria based on history taking. The patient claimed that he has no difficulties in passing urine and certainly had no significant bowel symptoms or change in bowel habits. Physical examination of the patient was essentially unremarkable and digital rectal examination did not reveal any abnormalities. Blood and urine investigations were normal except for microscopic haematuria with no evidence of urinary tract infection.

Radiological investigations including ultrasound and intravenous urogram demonstrated a left hydronephrosis with extrinsic compression of the bladder in the pelvis. Subsequently, a CT scan of abdomen and pelvis confirmed the extrinsic compression of bladder, rectum and sigmoid colon from pelvic fatty mass lesion (Figure 1). A T2 weighted $\mathrm{MRI}$ of the pelvis revealed the pelvic mass with high fatty tissue content, suggestive of pelvic lipomatosis (Figure 2). The patient was then counselled about the clinical scenario and decision was to perform a cystoscopy and retrograde left double J stenting to relief the obstructed left kidney. A formal rigid cystoscopy and cystogram (Figure 3) was performed, and it showed proliferative cystitis, elongation of the prostatic urethra and the elevation of the bladder neck. Biopsies of the florid cystitis bladder wall were taken for histopathological assessment.The biopsy of the bladder demonstrated cystitis glandularis.

Subsequently, a left percutaneous nephrostomy insertion was carried out with antegrade double J stenting to relief the obstruction. The patient then underwent exploratory laparotomy in hope of removing the perivesical fatty tumour mass and to ascertain the exact nature of the pelvic mass, which was causing the obstruction. Intraoperatively, the pelvic lesions were very adhered and inflamed with the dome of bladder noted at above the umbilicus region. Therefore partial
Correspondence:
$\mathrm{Ng}$ Keng Lim
Division of Urology, Department of Surgery, Faculty of Medicine, University of Malaya 50603 Kuala Lumpur, Malaysia
Email: kenglimng@yahoo.com 
excision of pelvic lesions and mobilization of bladder were carried out. The mobilisation of the left ureter was not possible due to extensive pelvic scarring and inflammation. The patient recovered well following the operation.

The histopathological analysis confirmed the presence of mature adipocytes with fibrous tissue consistent with pelvic lipomatosis. Since then the patient has remained asymptomatic and required a change of left double J stent. The further management of this benign condition has been extensively discussed with the patient and he is currently not keen for any further pelvic operations, but has promised to remain in our long term follow up for his condition.

\section{Discussion}

Pelvic lipomatosis is a benign overgrowth of adipose tissue with small amount of inflammatory and fibrotic

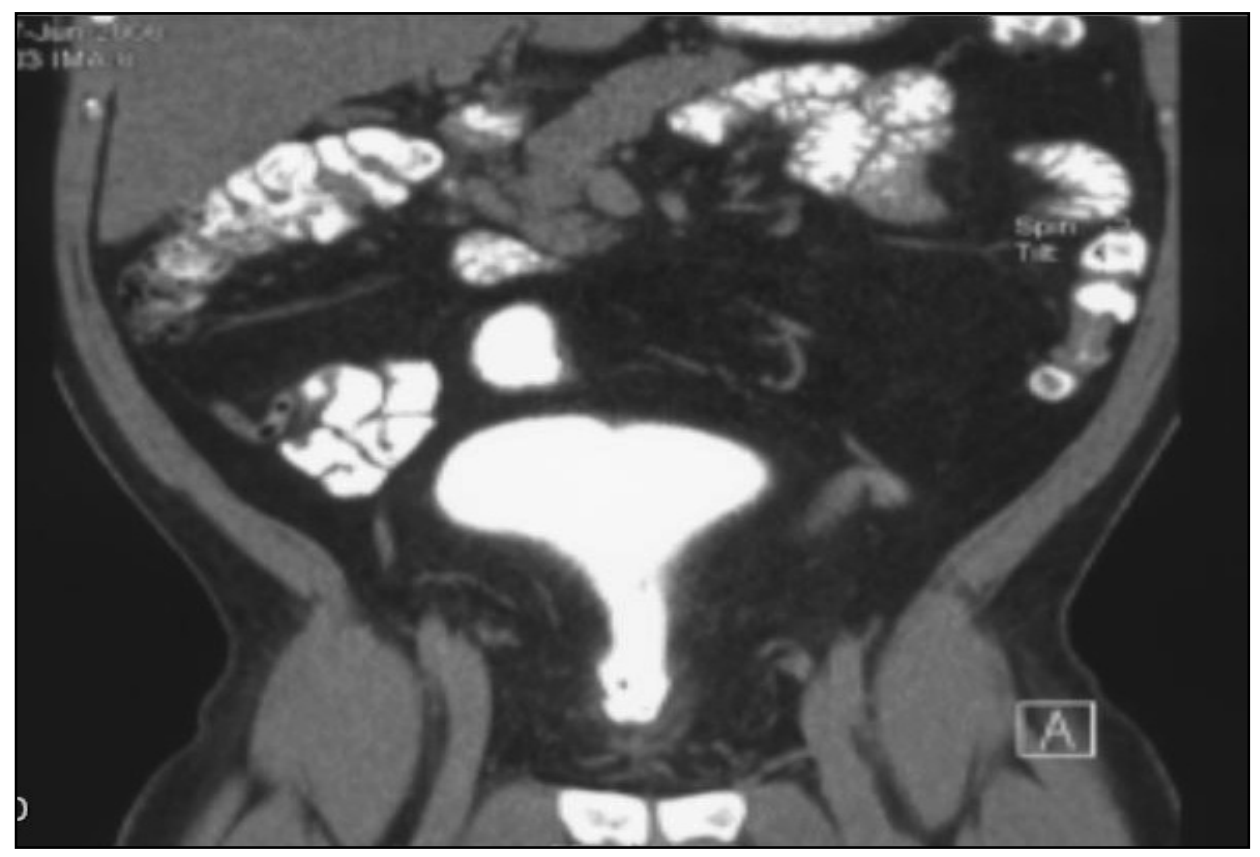

Figure 1: CT scan pelvis

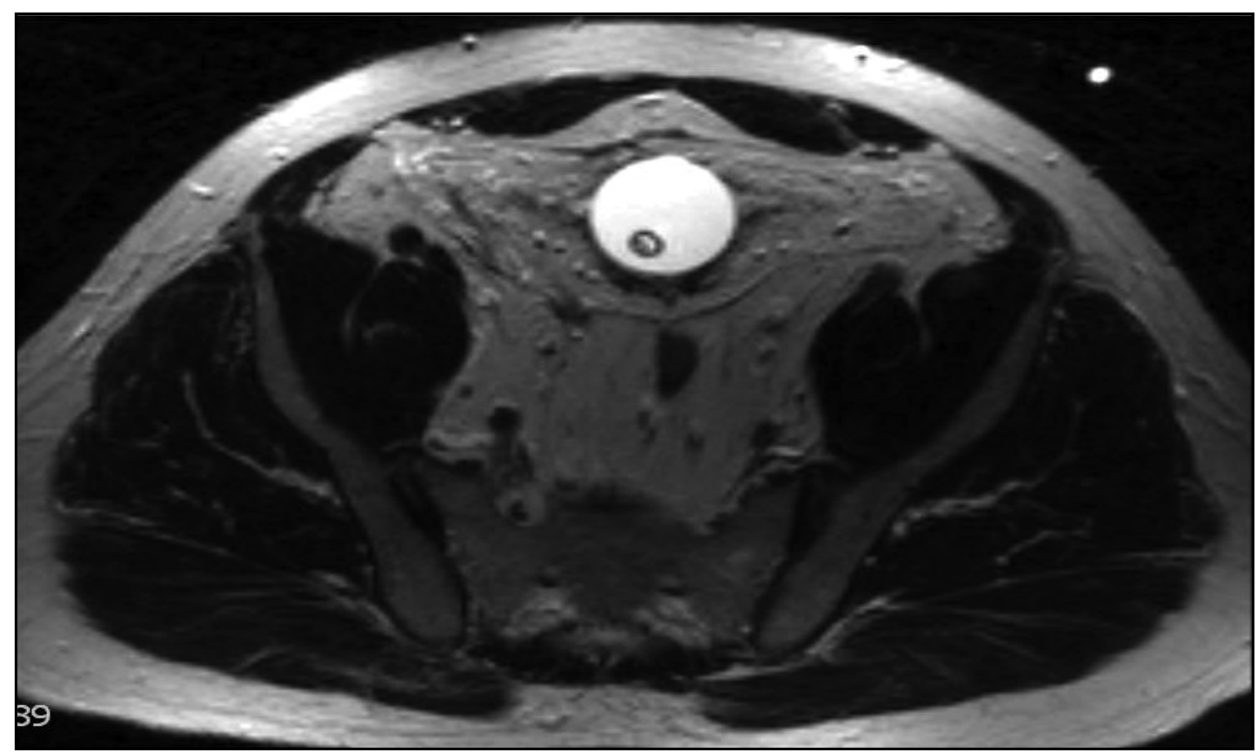

Figure 2: MRI (T2 weighted) scan of pelvis 


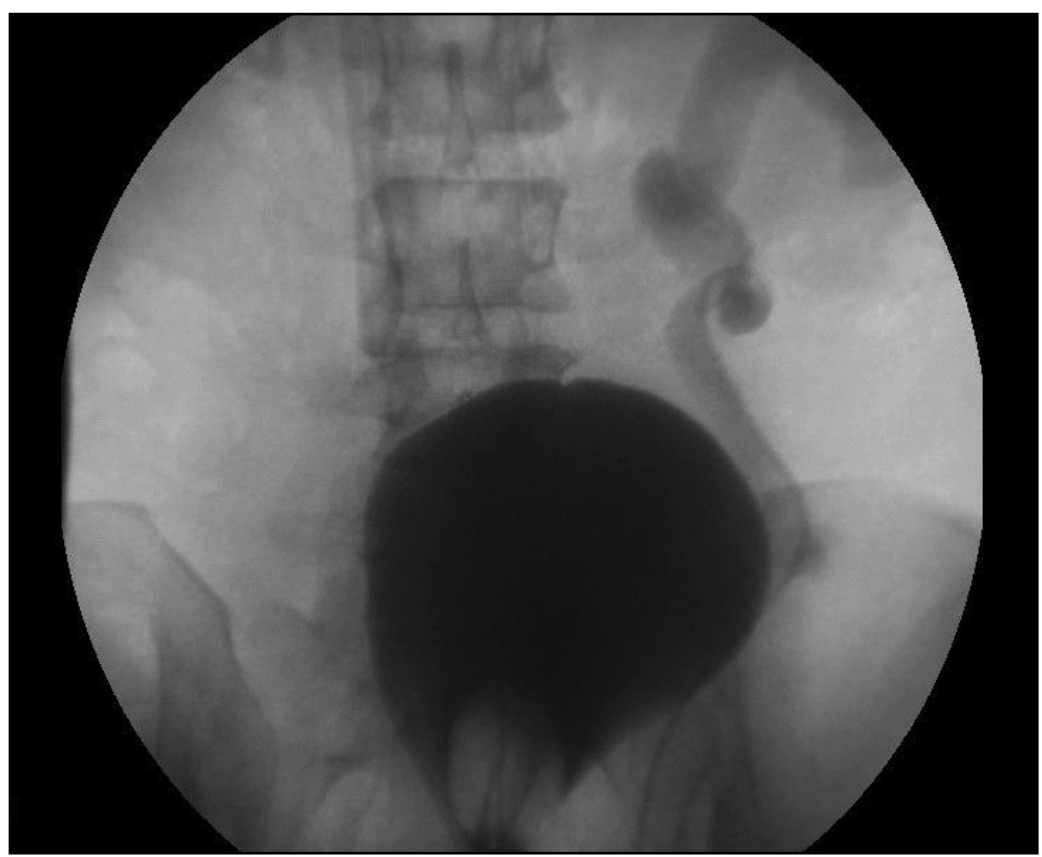

Figure 3: Cystogram

components found especially in the perivesical and perirectal spaces. Subsequently, it causes extrinsic compression of soft tissue structures in the pelvic cavity leading to its varied presentaions. It was first described by Engels, who reported five patients who had marked deformities of pelvic viscera, in 1959 (1). Peak incidence of this disease is between 25 to 60 years of age with a staggering male predominance of 10:1, and this disease is commonly noted in African Americans. The symptoms present include lower urinary tract symptoms, suprapubic pain, recurrent urinary tract infections, flank pain or even obstructive uropathy and bowel symptoms, which include tenesmus, constipation with oedema of lower extremities.

It can be found incidentally like in this case report following a routine health screening and contrary to popular belief, it has no correlation with obesity. Despite the benign nature of this disease, there is a risk of obstruction with hydronephrosis of $40 \%$ within five years (2). Radiological investigations usually reveal classical findings, which usually clinch the diagnosis even before tissue diagnosis and confirmation. A CT scan usually shows characteristic features of extensive fibrofatty proliferation of pelvic fat and tear shaped appearance of bladder. An IVU will show inverted tear drop shaped bladder caused by extrinsic compression with elevation of bladder base and dilated tortous medially or laterally displaced ureters.
Although no definite aetiological cause of the disease has been agreed upon, there are several theories postulated. These include endocrine dysfunction associated with diabetes milletus or Cushing's disease, chronic urinary tract infections causing fat redistribution in pelvis and obesity (24). However, in the management of this disease, most urologists will generally adopt a conservative approach with the use of symptomatic therapy and follow up for cases with minor symptoms and no renal impairment. Certain modalities of treatment have been employed with little success and these include long term antibiotics, steroids, diet control and even radiotherapy. Occasionally, an upper tract urinary diversion is required for those with obstructive uropathy with worsening renal function and severe symptoms. It has been well described that excision of the pelvic fat is difficult and time consuming but not impossible and response to surgery is good, but recurrence is possible (5). Ultrasonic assisted lipectomy and reimplantation of ureters have been employed successfully in cases of obstructive pelvic lipomatosis (6).

Most cases demonstrate slow progression with non specific symptoms. Because of that, the presentation of symptoms is often delayed, and it is difficult to predict which patient will deteriorate in terms of renal function and severity of symptoms. These patients are often lost to follow up. Therefore, most authors 
recommend that follow up is needed as future urinary diversion may be required for those who develop renal impairment secondary to obstructive uropathy. Interestingly, proliferative cystitis (eg, cystitis cystica, cystitis glandularis) are found in $76 \%$ of cases of pelvic lipomatosis, such as the case we described above. This proliferative pelvic lipomatosis disease can cause bladder outflow obstruction leading to proliferative cystitis and thus close follow up is needed because of its association with adenocarcinoma of bladder (7).

\section{Conclusion}

This rare case of pelvic lipomatosis presented to us incidentally following a routine health checkup revealed classical radiological images which have been shown above. Despite the benign nature of this disease, close follow up and early intervention is recommended because of the possibilities of future obstructive uropathy, its association with proliferative cystitis and the propensity for recurrence of disease.

\section{References}

1. Engels EP. Sigmoid colon and urinary bladder in high fixation. Roentgren changes simulating pelvic tumour. Radiology 1959, 72: 419-422.
2. Golding $\mathrm{PL}$, Singh $\mathrm{M}$, Worthington $\mathrm{B}$. Bolateral ureteric obstruction caused by benign pelvic lipomatosis. British J of Surgery 1972; 59: 69-72.

3. Malter IJ, Omell GJ. Pelvic lipomatosis in a woman. A case report. Obstet Gynaecol 1971; 37:63-66

4. Moss AA, Clark RE, Goldberg HE. Pelvic lipomatosis. A roentgrenographic diagnosis. AJR Am J Roentrenol 1972; 115: 411-419.

5. Shah $T$, Visshana $K$, Arora $R$, et al. pelvic lipomatosisa rare aetiology of bilateral ureteric obstruction Indian J of Urology 2001; 18: 82-83.

6. Halamachi S, Moskiwitz B, Calderon $\mathrm{N}$ et al. The use of ultrasonic assisted lipectomy device for treament of obstructive pelvic lipomatosis. Urology 1996; 48: 128-130.

7. Sozen S, Gorocak S, Uzum N, et al. The importance of reevaluation in patients with cystitis glandularis associated with Pelvic lipomatosis. Urol Oncol 2004; 22(5): 428. 